\title{
Suspension-adapted Chinese hamster ovary-derived cells expressing green fluorescent protein as a screening tool for biomaterials
}

\author{
E.-M. Engelhardt $\cdot$ S. Houis · T. Gries • \\ J. Hilborn · M. Adam · F. M. Wurm
}

Received: 27 January 2009/Revised: 10 March 2009/Accepted: 20 March 2009/Published online: 10 April 2009

(C) Springer Science+Business Media B.V. 2009

\begin{abstract}
Synthetic biomaterials play an important role in regenerative medicine. To be effective they must support cell attachment and proliferation in addition to being non-toxic and non-immunogenic. We used a suspension-adapted Chinese hamster ovary-derived cell line expressing green fluorescent protein (GFP) to assess cell attachment and growth on synthetic biomaterials by direct measurement of GFP-specific fluorescence. To simplify operations, all cell cultivation steps were performed in orbitallyshaken, disposable containers. Comparative studies between this GFP assay and previously established cell quantification assays demonstrated that this novel approach is suitable for rapid screening of a large number of samples. Furthermore the utility of our assay system was confirmed by evaluation of cell growth on three polyvinylidene fluoride polymer scaffolds that differed in pore diameter and drawing conditions. The data presented here prove the general
\end{abstract}

E.-M. Engelhardt · M. Adam · F. M. Wurm ( $\square)$ Laboratory of Cellular Biotechnology, Ecole Polytechnique Fédérale de Lausanne, School of Life Sciences, 1015 Lausanne, Switzerland

e-mail: florian.wurm@epfl.ch

\section{S. Houis · T. Gries}

Institut für Textiltechnik, RWTH Aachen University, Eilfschornsteinstrasse 18, 52062 Aachen, Germany

\section{J. Hilborn}

Department of Materials Chemistry, Polymer Chemistry, Uppsala University, 75121 Uppsala, Sweden utility of GFP-expressing cell lines and orbital shaking technology for the screening of biomaterials for tissue engineering applications.

Keywords Cell attachment - Cell growth . Green fluorescent protein - Scaffolds .

Screening $\cdot$ Suspension cells

\section{Introduction}

Tissue engineering scaffolds are needed to provide a temporary structure at the site of injury or disease to support cell attachment and growth along with synthesis of extracellular matrix proteins to eventually achieve the in vivo generation of a functional tissue. Determination of mechanical and physical properties is important for the characterization of scaffolds (O'Brien et al. 2005; Pattison et al. 2005), but currently in vitro scaffold-cell interactions are also frequently used to evaluate the bioactivity and cytotoxicity of a given material. For this purpose, the continuous monitoring of the number of viable cells attached to the scaffold is essential. Unfortunately, conventional cell enumeration techniques (i.e. direct cell counting, dye uptake, and DNA staining) are not suitable to assess cell growth on and within three-dimensional scaffolds. Scanning and transmission electron microscopy (SEM and TEM) are sometimes used to demonstrate cell proliferation on complex scaffolds but these time-consuming and labor-intensive techniques 
remain unsuitable in cases of multiple-parameter material screening, moreover these methods are not quantitative (Conconi et al. 2006).

To address this problem, we developed a cellbased assay for the screening of biomaterials using green fluorescent protein (GFP) as a surrogate marker for cell numbers. GFP has been used as a reporter protein and monitoring tool in many biological studies (Hunt et al. 1999; March et al. 2003). It does not require specific cofactors, its fluorescence is very stable and it can be expressed in many cell types (Kain and Chalfie 1998). Furthermore, GFP-specific fluorescence is a quantitative measure of the intracellular level of the protein in single cells ( $\mathrm{Li}$ et al. 2000). GFP quantification is feasible both in vitro and in vivo.

In the work described here we demonstrated the utility of suspension-adapted GFP-expressing $\mathrm{CHO}$ cells as a tool for screening biomaterials in vitro. The cells were maintained in suspension by orbital shaking rather than stirring. Even with agitation of the culture, the cells efficiently attached to the scaffold. Direct monitoring of cell attachment on three-dimensional structures by measuring GFP-specific fluorescence was demonstrated on poly(lactic acid-co- $\varepsilon$-caprolactone) (PLAC) polymer scaffolds. The GFP-based assay was validated by comparison with standard methods of cell quantification, and then applied to characterize the impact of different fiber structures of polyvinylidene fluoride (PVDF) scaffolds on cell attachment and cell growth.

\section{Materials and methods}

Cell culture

Suspension-adapted Chinese hamster ovary cells (CHO DG44) were cultivated in ProCHO5 medium (Lonza Verviers SPRL) as previously described (De Jesus et al. 2003; Muller et al. 2005).

\section{Plasmid DNA}

pMYKEF1-EGFP-puro was described previously (Derouazi et al. 2006). Plasmid DNA was purified on a Nucleobond AX anion exchange column (Macherey-Nagel, Düren, Germany) according to the manufacturer's protocol. Plasmids were linearized prior to transfection using $P v u \mathrm{I}$.

Generation of stable cell lines

Transfection of CHO DG44 cells in CultiFlask 50 tubes (Sartorius AG, Göttingen, Germany) was performed in $5 \mathrm{ml}$ ProCHO5 medium containing $2 \times 10^{6}$ cells $/ \mathrm{ml}$. Polyethylenamine (PEI) was used for plasmid DNA delivery as described previously (Derouazi et al. 2004). One day post-transfection, $10 \mu \mathrm{g}$ puromycin/ml was added daily for 10 days. Clonal puromycin-resistant cells were recovered by limiting dilution.

Scaffolds

PLAC was prepared as described (Ananta et al. 2009) and the polymer was melt spun into round multifilaments consisting of 25 filaments at G. Krahmer GmbH (Buchholz, Germany). Subsequently the PLAC yarns were processed by the circular knitting machine TK83 (HARRY LUCAS Textilmaschinen GmbH\&Co KG, Neumünster, Germany) with a needle gauge of E28.

PVDF SOLEF 1006 (Solvay Solexis S.A.S., Tavaux, France) was spun at the Institut für Textiltechnik (RWTH Aachen University, Germany) to trilobal multifilaments consisting of 24 filaments. A customdesigned double raschel warp knitting machine (Typ DR 16 EEC/EAC, Karl Mayer Textilmaschinenfabrik $\mathrm{GmbH}$, Obertshausen, Germany) was used for the production of three-dimensional polymeric scaffolds at a needle gauge of E30. The fibers were pulled individually from the bobbins. Knitting settings were kept constant for all PVDF batches to ensure that scaffold structure did not affect cell growth.

All polymer scaffolds were cut into discs of $1 \mathrm{~cm}^{2}$ and sterilized in $70 \%(\mathrm{v} / \mathrm{v})$ ethanol.

Cell seeding on scaffolds

Several sterilized scaffold discs were added to a CultiFlask 50 tube containing a $10 \mathrm{ml}$ culture of CHO-GFP4 cells at $0.3 \times 10^{6}$ cells $/ \mathrm{ml}$ (culture A) in ProCHO5 medium supplemented with $0.5 \%$ fetal bovine serum (FBS). After $4 \mathrm{~h}$ incubation with agitation at $180 \mathrm{rpm}$ (seeding phase), the scaffold discs in each tube were transferred to a new CultiFlask 
50 tube with $10 \mathrm{ml}$ fresh ProCHO 5 medium supplemented with $0.5 \%$ FBS (culture B). Scaffolds from cultures A and B were used to measure cell attachment and growth of attached cells, respectively.

\section{GFP assay}

At various time points, one scaffold disc and $1 \mathrm{ml}$ culture medium were removed from each CultiFlask 50 tube. The culture medium was transferred into a 24-well plate and centrifuged for $5 \mathrm{~min}$ at $1,500 \times \mathrm{g}$. The supernatant was discarded and the cells in each well were lysed by addition of $1 \mathrm{ml}$ lysis buffer (1\% Triton X-100 in PBS). Scaffold discs were transferred to a 24-well plate, washed once in PBS, and the attached cells were lysed by addition of $1 \mathrm{ml}$ lysis buffer. After $1 \mathrm{~h}$ at $37^{\circ} \mathrm{C}$ with agitation, $200 \mu \mathrm{l}$ cell lysate was transferred to a 96-well plate and the GFPspecific fluorescence was measured using a Safire $^{2}$ Microplate Reader (excitation wavelength: $485 \mathrm{~nm}$; emission wavelength: $515 \mathrm{~nm}$ ) (Tecan AG, Männedorf, Switzerland).

\section{Alamar Blue assay}

Cell-seeded scaffold discs seeded were transferred to a 24-well plate, washed with PBS, and the Alamar Blue assay was performed according to the manufacturer's protocol.

\section{PicoGreen assay}

Scaffold discs from culture A were treated with DNase I (Roche) at the end of the seeding phase according to manufacturer's protocol. After three washes in PBS, genomic DNA was extracted from the scaffolds using a standard protocol. DNA extraction was also performed with $1 \mathrm{ml}$ of cell culture. After centrifugation at $800 \times g$ for $5 \mathrm{~min}$, genomic DNA was extracted from the cell pellet. Genomic DNA was quantified using the Quant-iT PicoGreen assay kit (Invitrogen).

Scanning electron microscopy (SEM)

Cell-seeded polymers were fixed in $0.25 \%$ glutaraldehyde at room temperature for $1 \mathrm{~h}$ and then dehydrated in a series of graded ethanol washes (50\% to $100 \%$ ethanol). Polymers were further dried by supercritical $\mathrm{CO}_{2}$ extraction (5 times for $5 \mathrm{~min}$ and once for $20 \mathrm{~min}$ ). Samples were coated with $20 \mathrm{~nm}$ platinum and examined by SEM at $5 \mathrm{kV}$ (JEOL 6300F, JEOL, Tokyo, Japan).

\section{Results}

Characterization of GFP-expressing CHO cells

The GFP expression level of CHO-GFP4 cell line was stable over 2 months in culture in the absence of selective pressure. All the cells were GFP-positive with a mean expression of 820 Relative Fluorescent Units (RFU) when cultured in suspension. The GFPspecific fluorescence of CHO-GFP4 also correlated with cell number up to $4 \times 10^{6}$ cells (Fig. 1). The stable GFP expression level over time and the direct relation between the GFP-specific fluorescence and the cell number make GFP-expressing cells suitable as a screening tool for biomaterials.

\section{Scaffold characterization}

Some characteristics of the PLAC and PVDF fibers used in this study are provided in Table 1. Trilobal

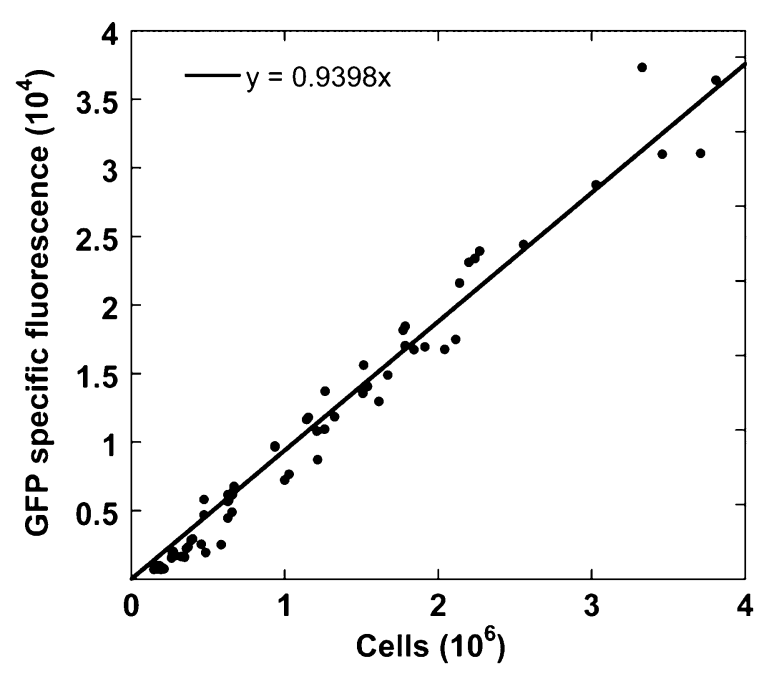

Fig. 1 Correlation between GFP-specific fluorescence and cell number for a given cell line. The number of CHO-GFP4 cells was plotted against the GFP-specific fluorescence. The linear standard curve shows that the GFP-specific fluorescence is directly related to the cell number. Data are based on three independent experiments each containing triplicates 
Table 1 Main characteristics of PLAC and PVDF fibers

\begin{tabular}{lllcrr}
\hline Sample & No of filaments & Draw ratio & Yarn count (dtex) ${ }^{\mathrm{a}}$ & $\begin{array}{l}\text { Tensile strength } \\
\text { at break (cN/dtex) }\end{array}$ & $\begin{array}{l}\text { Elongation } \\
\text { at break (\%) }\end{array}$ \\
\hline PLAC & 25 & 1 & $151.7 \pm 3.81$ & $13.48 \pm 0.98$ & $111.38 \pm 15.12$ \\
PVDF H & 24 & 1 & $337.57 \pm 0.64$ & $10.8 \pm 0.7$ & $195.03 \pm 13.09$ \\
PVDF L & 24 & 2.5 & $257.63 \pm 0.10$ & $21.3 \pm 1.1$ & $90.88 \pm 8.35$ \\
PVDF N & 24 & 1 & $181.51 \pm 4.63$ & $17 \pm 1.1$ & $129.23 \pm 8.74$ \\
\hline
\end{tabular}

The linear density of the fibers was determined according to DIN EN ISO 2060, and tensile tests $(n=30)$ were performed according to DIN 53834

a Yarn count defines the quantity of polymer in grams per $10 \mathrm{~km}$ (dtex units)

Table 2 Average pore areas of PLAC and PVDF scaffolds

\begin{tabular}{ll}
\hline Scaffolds & Average pore area $(\mu \mathrm{m})$ \\
\hline PLAC & $213.70 \pm 62.30$ \\
PVDF H & $142.00 \pm 54.14$ \\
PVDF L & $149.60 \pm 64.62$ \\
PVDF N & $213.80 \pm 89.93$ \\
\hline
\end{tabular}

Porosity and pore size of the scaffolds were measured using image analysis with MATLAB in combination with an optical microscope

PVDF fibers with different yarn counts and morphologies were produced to investigate the influence of the fiber structure on cell attachment and growth. Different morphologies were obtained by drawing sample L (draw ratio 2.5) but not samples $\mathrm{H}$ and $\mathrm{N}$ (draw ratio 1). Without drawing the fiber had less strength and greater elongation. Drawn fibers also have a rougher surface than non-drawn fibers (Jee et al. 2007) The average pore size and pore areas of PLAC and PVDF scaffolds are presented in Table 2. Sample $N$ had larger pore size than the other two PVDF scaffolds due to its lower yarn count (Table 1).
Cell attachment on PLAC scaffolds

Scaffold discs were incubated in an orbitally shaken suspension culture of CHO-GFP4 cells (culture A). Cultivated mammalian cells are known to adhere to suspended particles as demonstrated in bioprocesses using microcarrier beads in stirred vessels (Cahn 1990). Here orbital shaking rather than stirring was used as mixing principle because this allows maintenance of single-suspension cultures with adequate gas transfer and it ensures that no impeller or baffles would damage the scaffolds or interfere with cell attachment. All cell cultivations were performed in a medium that contained factors promoting cell attachment like serum and calcium.

CHO-GFP4 cells attached to PLAC scaffolds within $4 \mathrm{~h}$ of incubation with agitation. Attached cells were visualized within the scaffold using conventional fluorescence microscopy in a non-invasive way (Fig. 2a). Attached cells had either a flat, spindle shaped morphology (Fig. 2b) or were more rounded with many filopodia (Fig. 2c). Cell attachment efficiency was determined after an incubation period of
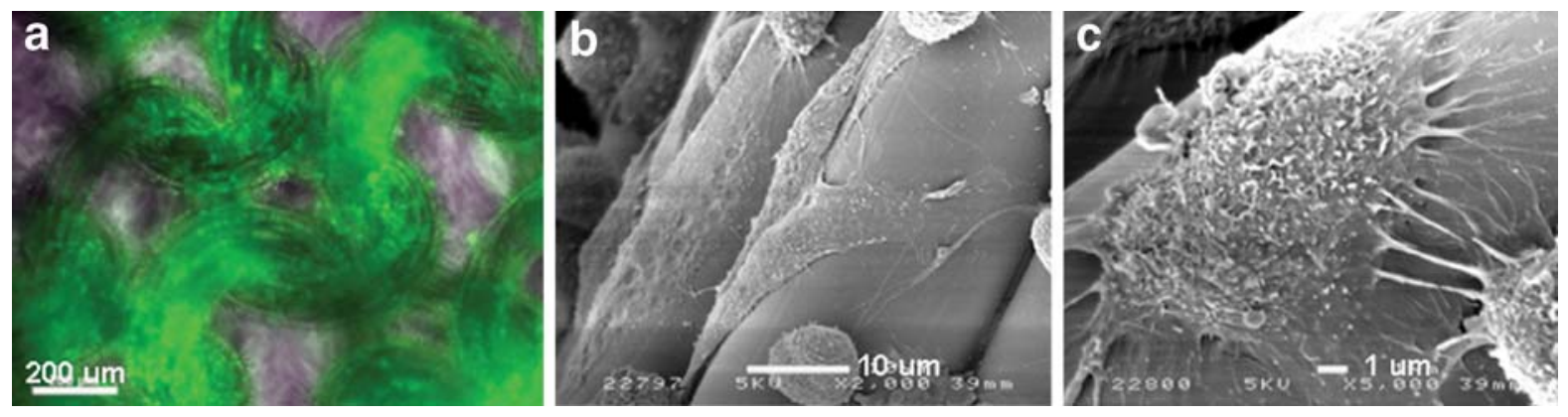

Fig. 2 CHO-GFP4 cells attached onto a PLAC scaffold on day 2 visualized by fluorescence light microscopy (a) and SEM (b, c). Attached cells show either a flat, spindle-shaped morphology (b) or a round- shaped morphology (c). Attachment on PLAC scaffolds was performed in CultiFlask 50 tubes incubated in a shaker with orbital agitation at $180 \mathrm{rpm}$. Seal bars are $200 \mu \mathrm{m}(\mathbf{a}), 10 \mu \mathrm{m}(\mathbf{b})$ and $1 \mu \mathrm{m}(\mathbf{c})$ 


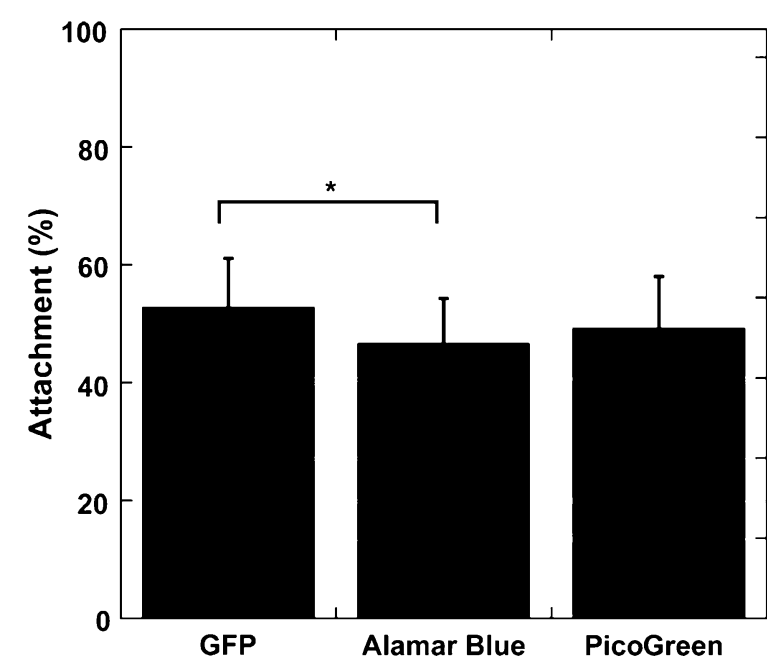

Fig. 3 Percentage of cell attachment onto PLAC scaffolds at the end of the seeding phase $(4 \mathrm{~h})$. Attachment was calculated based on the GFP ( $n=5,6$ samples per experiment), Alamar Blue ( $n=5,6$ samples per experiment) and Pico Green assays ( $n=1,6$ samples). Kruskal and Tukey test (software R2.7.1) showed $P=0.035$ when comparing GFP and Alamar Blue assays groups

$4 \mathrm{~h}$ using three different fluorescence-based assays: the GFP, Alamar Blue, and PicoGreen assays. With each assay, the fluorescence associated with attached cells was compared to the total cell fluorescence (attached plus suspension cells). The percentage of cell attachment was found to be about $50 \%$ for each of the individual assays (Fig. 3). Statistical analyses (Kruskal and Tukey test, software R2.7.1) indicated that the difference observed between the GFP- and Alamar Blue-based assays was significant (Kruskal test, $P=0.035)$. Since the Alamar Blue-based assay reflects cellular metabolism which might be different between adherent and suspension cells, we devalued the result of the Kruskal test. Furthermore, the $P$-value was close to the decision limit. Based on these results, it was concluded that the GFP-specific fluorescence assay was appropriate to determine the level of cell attachment on PLAC scaffolds.

\section{Cell growth on PLAC scaffolds}

The GFP assay was also used to monitor cell growth on PLAC scaffolds. At the end of the seeding phase, scaffold discs were transferred to agitated cultivation tubes containing fresh medium without cells (culture B). This transfer ensured that only cells attached to

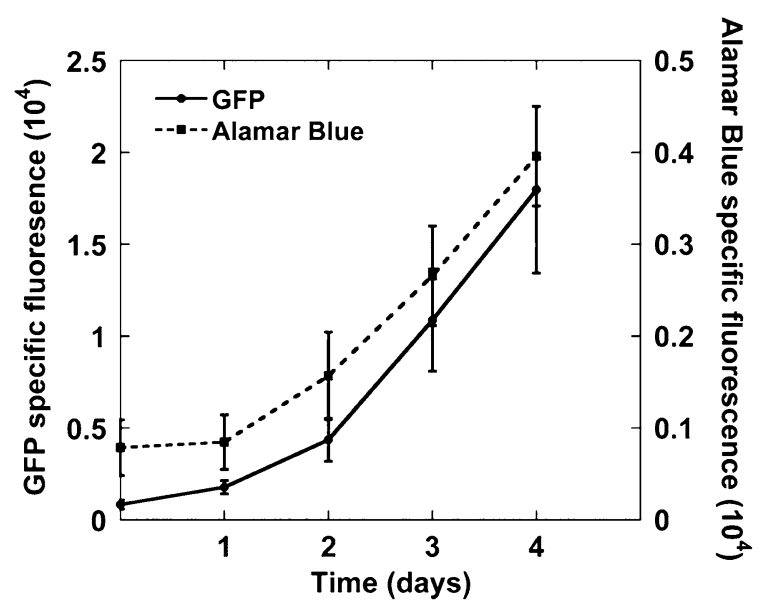

Fig. 4 Growth of CHO-GFP4 cells on PLAC scaffolds monitored over a period of 4 days using either the GFP ( $n=2,6$ samples per experiment) or the Alamar Blue assay ( $n=2,6$ samples per experiment). Both methods revealed similar trends regarding growth pattern and calculated cell numbers on the scaffolds at day 4

the scaffold discs would be measured. The growth of scaffold-attached cells was monitored over a 4-day period using both the GFP- and Alamar Blue-based assays. The growth curves derived from the data obtained by the two methods were similar (Fig. 4). Conversion of the fluorescence measurements into cell number was accomplished using the appropriate standard curves of GFP-specific (Fig. 1) and Alamar Blue-specific fluorescence (data not shown). On day 4 of the culture, the GFP- and Alamar Blue-based assays measured $1.9 \pm 0.4 \times 10^{6}$ and $1.7 \pm 0.2 \times 10^{6}$ attached cells, respectively.

Cell growth on PVDF scaffolds

As physical and structural properties of scaffolds influence the cellular response (O'Brien et al. 2005; Moroni et al. 2006), PVDF scaffolds with different fiber structures were fabricated and then evaluated using CHO-GFP4 cells. Cell attachment and growth of the attached cells was determined by measuring GFP-specific fluorescence associated with scaffold discs over a 4-day period. The three PVDF scaffolds used in this experiment differed in pore diameter and fiber morphology (Table 2). Measurement of GFPspecific fluorescence over time revealed differences between the three scaffolds in terms of their support of cell growth (Fig. 5). The slightly different number 


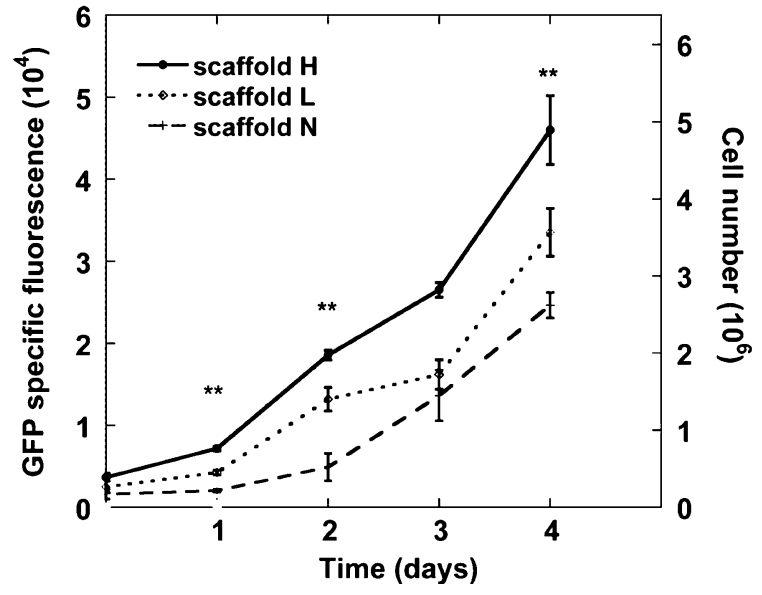

Fig. 5 Growth evaluation of CHO-GFP4 cells on three different PVDF scaffolds $(n=3)$ over a period of 4 days. Statistical analyses (software R2.7.1) revealed significant differences $(* * P<0.01)$ of the number of attached cells on the three scaffolds. Scaffolds $\mathrm{N}$ and $\mathrm{H}$ had the same fiber morphology (draw ratio 1) but differed in pore diameter. Therefore the difference in terms of growth might be due to the pore size. PVDF scaffolds $\mathrm{H}$ and $\mathrm{L}$ had similar pore diameters, but fibers in scaffold $\mathrm{L}$ were drawn resulting in a smoother surface

of attached cells on day 0 was due to the differences in cell attachment on the three PVDF scaffolds during the seeding phase. Considering conversion of GFP-specific fluorescence into cell number using the standard curve (Fig. 1), the highest cell number at day 4 was associated with scaffolds $\mathrm{H}\left(4.8 \pm 0.5 \times 10^{6}\right.$ cells $)$; intermediate cell number emerged on scaffolds $\mathrm{L}$ $\left(3.5 \pm 0.3 \times 10^{6}\right.$ cells $)$ and the lowest on scaffolds $\mathrm{N}$ $\left(2.6 \pm 0.3 \times 10^{6}\right.$ cells $)$. These data demonstrated that the PVDF scaffolds with the largest average pore diameter (scaffolds $\mathrm{N}$ ) were the poorest for supporting cell growth. The two other PVDF scaffolds (H and L) had similar average pore diameters but differed in terms of fiber morphology (Table 2). Scaffolds $\mathrm{H}$ made of non-drawn trilobal PVDF fibers provided better support for cell growth than the drawn scaffolds L.

\section{Discussion}

This study demonstrated the utility of suspensionadapted GFP-expressing CHO cells to screen biomaterials for tissue engineering purposes. The attachment assay was validated by direct comparison with established cell quantification techniques such as the
Alamar Blue assay and PicoGreen-based DNA quantification The monitoring of GFP-expressing cells on three-dimensional structures for cell attachment and growth was achieved using PLAC and PVDF textile scaffolds. An important and necessary feature of the screening assay is the ability of CHO-GFP4 cells to switch from suspension to adherent growth. The availability of suspension-adapted cells makes the scale-up of cell number relatively simple. As a consequence, this screening method offers the possibility of testing several hundred samples and/or culture conditions in high-throughput experimental set-ups with cells from a single culture. In addition, cell cultivation in suspension cultures drastically simplifies the procedure as no treatment with trypsin is required to recover cells prior to their use in the cell attachment assay with the polymers.

Our proposed screening system presents several advantages compared to the Alamar Blue and the PicoGreen assay. The latter is unsuitable for high throughput experiments because of the labor-intensive DNA extraction step in contrast to the GFP and Alamar Blue assays. The Alamar Blue assay is based on the quantification of the cellular metabolic activity through the reduction of a non-fluorescent substrate to a fluorescent product. Inaccuracies in the assay may occur if the metabolic activity changes along with the cell density on the scaffold. Furthermore, the incubation time with Alamar Blue needs to be accurately assessed in a cell-dependent way to avoid saturation of the reaction, meaning $100 \%$ conversion of the non-fluorescent substrate into the fluorescent product. Finally, the Alamar Blue assay relies on the diffusion of the dye to cells within the scaffolds. However, some cells may be inaccessible to the dye, resulting in an underestimation of the cell number. The GFP assay ensures accurate quantification of cells entrapped in the scaffold since the reporter protein is expressed intracellularly and the amount of GFP per cell is constant within the clonal cell population. Another important advantage of our assay is the possibility of visualizing GFP-expressing cells on and within scaffolds using fluorescence microscopy. This allows qualitative, non-invasive examination of polymeric scaffolds prior to the quantification of cell number.

The analysis of three different PVDF scaffolds for cell growth showed that cellular responses to different scaffold parameters can be revealed and quantified 
using GFP-expressing cells. Hence our assay showed that cell growth was better on PVDF scaffolds with small pore areas, confirming previously published results (O'Brien et al. 2005). A reduced number of cell-anchor points on scaffolds with large pore areas is expected to result in poor cell attachment and growth. Enhanced cell growth on scaffolds $\mathrm{H}$ relative to $\mathrm{L}$ indicated that fiber morphology also plays a role in cell attachment and growth. The lack of fiber drawing in scaffolds $\mathrm{H}$ resulted in less aligned macromolecular polymer chains and thus in a lower crystallinity compared to the fibers in scaffolds L. Crystal structures influence the surface properties of PVDF materials (Lovinger 1982), thus affecting cell behavior. The study described here is a proof of principle for the applicability of GFP-expressing cells to the screening of biomaterials. Although a CHO-derived cell line was employed for the studies here, it is conceivable that GFP-expressing primary cells could also be used to screen potential scaffold materials. Current DNA delivery methods like nucleofection (Hamm et al. 2002) or viral gene delivery (Durual et al. 2007) allow the efficient transfection of different cell types, particularly primary cells.

The application of suspension-adapted $\mathrm{CHO}$ cells in scaffold attachment assays has not been reported previously. It was possible to perform the assay in suspension culture because we used orbital shaking rather than stirring as a mixing principle. We verified the observations made with GFP-expressing $\mathrm{CHO}$ cells on different PVDF scaffolds using L929 fibroblasts. Similar results to those described here were obtained (E. Engelhardt and S. Houis, unpublished data), confirming our conclusions. Thus we believe that suspension-adapted GFP-expressing $\mathrm{CHO}$ cells are a valuable tool for characterizing cell-scaffold interactions.

Acknowledgments The authors would like to thank Dr. David Hacker and Dr. Lucia Baldi for their contribution in reviewing this manuscript and Antonio Mucciolo (Centre de Microscopie éléctronique, Faculté de Médcine et Biologie, Université de Lausanne, Switzerland) for his advice and help with the electron microscopy. This research was financed by EU-project 013602 3G-SCAFF.

\section{References}

Ananta M, Aulin C, Hilborn J et al (2009) A poly (lactic acid-cocaprolactone)-collagen hybrid for tissue engineering applications. Tissue Eng 15. doi: 10.1089/ten.tea.2008.0914

Cahn F (1990) Biomaterials aspects of porous microcarriers for animal cell culture. Trends Biotechnol 8(5):131-136

Conconi MT, Lora S et al (2006) In vitro evaluation of poly[bis(ethyl alanato)phosphazene] as a scaffold for bone tissue engineering. Tissue Eng 12(4):811-819

De Jesus M, Girard P et al (2003) TubeSpin satellites: a fast track approach for process development with animal cells using shaking technology. Biochem Eng J 17:217-223

Derouazi M, Girard P et al (2004) Serum-free large-scale transient transfection of $\mathrm{CHO}$ cells. Biotechnol Bioeng 87(4):537-545

Derouazi M, Martinet D et al (2006) Genetic characterization of CHO production host DG44 and derivative recombinant cell lines. Biochem Biophys Res Commun 340(4): 1069-1077

Durual S, Rideau A et al (2007) Lentiviral PU.1 overexpression restores differentiation in myeloid leukemic blasts. Leukemia 21(5):1050-1059

Hamm A, Krott N et al (2002) Efficient transfection method for primary cells. Tissue Eng 8(2):235-245

Hunt L, Jordan M et al (1999) GFP-expressing mammalian cells for fast, sensitive, noninvasive cell growth assessment in a kinetic mode. Biotechnol Bioeng 65(2):201-205

Jee T, Lee H et al (2007) Effect of microstructures of PVDF on surface adhesive forces. Tribol Lett 26:125-130

Kain S, Chalfie M (1998) GFP-green fluorescent protein; properties, applications and protocols. Wiley,

Li J, Wang S et al (2000) Green fluorescent protein in Saccharomyces cerevisiae: real-time studies of the GAL1 promoter. Biotechnol Bioeng 70(2):187-196

Lovinger AJ (1982) Developments in crystalline polymers. Applied Science Publications, London

March JC, Rao G et al (2003) Biotechnological applications of green fluorescent protein. Appl Microbiol Biotechnol 62(4):303-315

Moroni L, Licht R et al (2006) Fiber diameter and texture of electrospun PEOT/PBT scaffolds influence human mesenchymal stem cell proliferation and morphology, and the release of incorporated compounds. Biomaterials 27(28): 4911-4922

Muller N, Girard P et al (2005) Orbital shaker technology for the cultivation of mammalian cells in suspension. Biotechnol Bioeng 89(4):400-406

O'Brien FJ, Harley BA et al (2005) The effect of pore size on cell adhesion in collagen-GAG scaffolds. Biomaterials 26(4):433-441

Pattison MA, Wurster S et al (2005) Three-dimensional, nanostructured PLGA scaffolds for bladder tissue replacement applications. Biomaterials 26(15):2491-2500 